\title{
Mining Mill Condition Monitoring Using Water Jet Ultrasonics
}

\author{
Christer Stenström*, Johan E. Carlson ${ }^{\dagger}$, and Jan Lundberg* \\ *Div. of Operation and Maintenance Engineering \\ Luleå University of Technology \\ SE-971 87 Luleå, Sweden \\ $\dagger^{\dagger}$ Div. of Systems and Interaction \\ Luleå University of Technology \\ SE-971 87 Luleå, Sweden \\ Email: johan.carlson@ltu.se
}

\begin{abstract}
In this paper we investigate the feasibility of using a water jet ultrasound scanning system for condition monitoring of the shell of a rotating mining mill. The practical requirements of such a system were first analyzed, which shows that it is possible to achieve the spatial resolution of $1 \times 1 \mathrm{~mm}$, using a pulse-echo setup with a pulse repetition frequency of approximately $6 \mathrm{kHz}$.

Experiments were then performed on a lab-scale prototype, where typical defects were introduced. The results show that it is possible to detect and image internal defects down to a size of around than $1 \times 0.4 \mathbf{~ m m}$.
\end{abstract}

\section{INTRODUCTION}

Mining is a continuous process where maximum availability is desired. Mining mills are continuously exposed to wear and impact loads that give rise to wear of the liners, as well as fatigue and crack development in the steel shell of the mill. Stoppages due to corrective and preventive maintenance lead to large production losses and equally large monetary losses. Condition monitoring can assist in the maintenance planning and decision process to increase the availability and the overall performance of the material processing. The objective of the proposed ultrasonic technique, which is using a water jet to transmit the ultrasound to the mill shell, is to detect and localize cracks within the shell without stopping the mill.

Non-destructive testing (NDT) refers to non-invasive techniques used for characterization and inspection of objects. Important fields are aviation, automotive, construction, marine, nuclear, wind turbines, circuit boards, pipes and sport goods, among others. NDT is used for research, manufacturing monitoring and in-service inspections to extract information of the integrity of a material. Besides visual inspection, ultrasonic testing (UT) is one of the most commonly used NDT techniques, together with radiography and thermography.

Radiography is the use of an x-ray beam propagating through an object and taken up by a film or detector. The attenuation of the x-ray beam depends on the spatial density variations within the object, which is the basis for forming the image. Radiography is more sensitive than ultrasonics for finding transverse cracks, but on the other hand, planar defects perpendicular to the X-ray source can go undetected [1], [2]. Radiography requires two-sided access, has limited thickness capabilities, and it is hazardous. Therefore it is of interest to find another methods which are able to measure crack developments in mining mills.

Thermography is another method which is used in many industries. Both active and passive thermography techniques are used. In an active arrangement a heat pulse is applied to the surface of an object to raise the temperature by a few degrees. The heat dispersion and radiation at the surface is affected by anomalies near the surface. An image is constructed by the use of a thermography camera. The method is less capable of detecting small and subsurface discontinuities but it is rapid and the results are easy to interpret [2], [3]. Since the shell material of a mining mill is a couple of centimeters thick and therefore another method for NDT is needed.

Ultrasonic testing has high resolution, good penetration depth, one and two-sided set-up possibilities, and it is nonhazardous. The main drawback is its line-by-line scan which makes it relatively slow compared to some other NDT methods [4], [5]. Another drawback is that it is very hard to use UT for detecting cracks in course-grained materials due to diffuse scattering and attenuation [6], [7]. Moreover, ultrasonic inspection usually requires an experienced operator [8]. Nevertheless, it is a capable technique, and in this study we evaluate the feasibility of implementing such an UT system for condition monitoring of mining mills, during operation.

The theoretical limitations of the technique are first investigated in Section II-A, in relation to the constraints imposed by a full-scale practical system. Experiments in a laboratory environment on well-defined material specimens are then conducted. Section II-B describes the processing steps applied to the measured ultrasound pulses. A lab-scale prototype, with similar material characteristics as the steel shell of a mining mill, was constructed, and some typical defects were introduced to evaluate the performance of the ultrasonic scanning system. This setup is described in Section III. Finally, the processed ultrasound signals were used to create an image 
of the steel shell. These results are described in Section IV.

\section{THEORY}

\section{A. Feasibility of full-scale setup}

In this section we will consider the practical constraints involved in constructing an ultrasonic inspection system suitable for condition monitoring of a full-scale mining mill.

If amplitudes of reflected ultrasound waves are to be used to image the interior of the mill shell, the maximum achievable spatial resolution of this image (i.e. the C-scan) is limited by the circumferential velocity of the mill, the choice of transducer, and by the computer performance. Dandotiya et al. [9] provide data for two typical mills that are used here to calculate the tangential velocity at the mill shell periphery. Mill one has a diameter of $5.7 \mathrm{~m}$ and an operating angular velocity of $8 \mathrm{rpm}$. Mill two has a diameter of $10.6 \mathrm{~m}$ and operates at $10.4 \mathrm{rpm}$. The circumferential velocities are then given by

$$
v_{\text {Rot }}=\omega r=2 \pi r f,
$$

resulting in $2.4 \mathrm{~ms}^{-1}$ and $5.8 \mathrm{~ms}^{-1}$ for mill one and two, respectively. A pulser/receiver with a pulse repetition frequency (PRF) of about $6 \mathrm{kHz}$ or more is then needed for a circumferential resolution of $1 \mathrm{~mm}$ for the second mill in the example. A PRF of $6 \mathrm{kHz}$ corresponds to a time of $167 \mu \mathrm{s}$ between each pulse. The time of flight (TOF), from sent to received pulse, in a pulse echo setup is

$$
T O F=\left(2\left(l_{1} / v_{1}+l_{2} / v_{2}\right) .\right.
$$

The variables $l$ and $v$ are the thickness and the speed of sound in water and the body under test, respectively. The speed of sound in water is around $1480 \mathrm{~ms}^{-1}$ and the longitudinal speed of sound in steel is around $5900 \mathrm{~ms}^{-1}$, at room temperature [10].

The minimum length of the water jet, i.e. the minimum distance between the transducer and the mill shell depends on several factors. For example bolt joints in the shell prevents the transducer from being positioned too close to the mill shell. The thickness of the shell is normally a couple of centimeters. As an example for calculation of $T O F$, with a water jet length of five centimeters and a shell thickness of three centimeters, the $T O F$ is then $2(50 / 1.48+30 / 5.9) \mu \mathrm{s}=78 \mu \mathrm{s}$, which is shorter than the time between each pulse, $167 \mu \mathrm{s}$. With a circumferential resolution of $1 \mathrm{~mm}$, it can then be suitable to have a resolution along the rotation axis of the mill of the same order, giving an overall spatial resolution of $1 \times 1 \mathrm{~mm}$.

The calculations above suggest that it is feasible to construct a system able to perform the inspection with the desired resolution.

One remaining issue is the total scanning time. For the first mill in the example above, one turn of the mill takes $7.5 \mathrm{~s}$. If the mill is $5 \mathrm{~m}$ long, and we want to measure every millimeter along axis of the mill, performing a full scan would take approximately $10.5 \mathrm{~h}$, not taking into account any time required for computation, moving the transducer, or storing data. This might seem like a prohibitively long time, but it would still enable at least one full scan per day of operation.

\section{B. Signal processing}

The signals from the water jet ultrasound scanning system, operating in pulse-echo mode (described in more detail in Section III), were sampled using a sampling frequency of $f_{s}=200 \mathrm{MHz}$, so that the digitized version $x_{0}(n)=x_{0}\left(n T_{s}\right)$, where $T_{s}=1 / f_{s}$. To reduce the storage requirement and the data processing time, the pulses were then down-sampled a factor of five, so that the resulting signals, $x(n)$ corresponded to pulses sampled at $40 \mathrm{MHz}$.

The envelopes of the measured ultrasound pulses (A-scans) were computed as [11]

$$
y(n)=|x(n)+j \mathcal{H}\{x(n)\}|,
$$

where $\mathcal{H}\{x(n)\}$ denotes the discrete Hilbert transform of $x(n)$. A 7th order Butterworth low-pass filter with a cutoff frequency of $0.15 \mathrm{rad} / \mathrm{s}$ (normalized frequency) was then applied to $y(n)$ to further suppress the oscillations caused by the ultrasound pulse shape. The result of this operation is shown in Fig. 1.
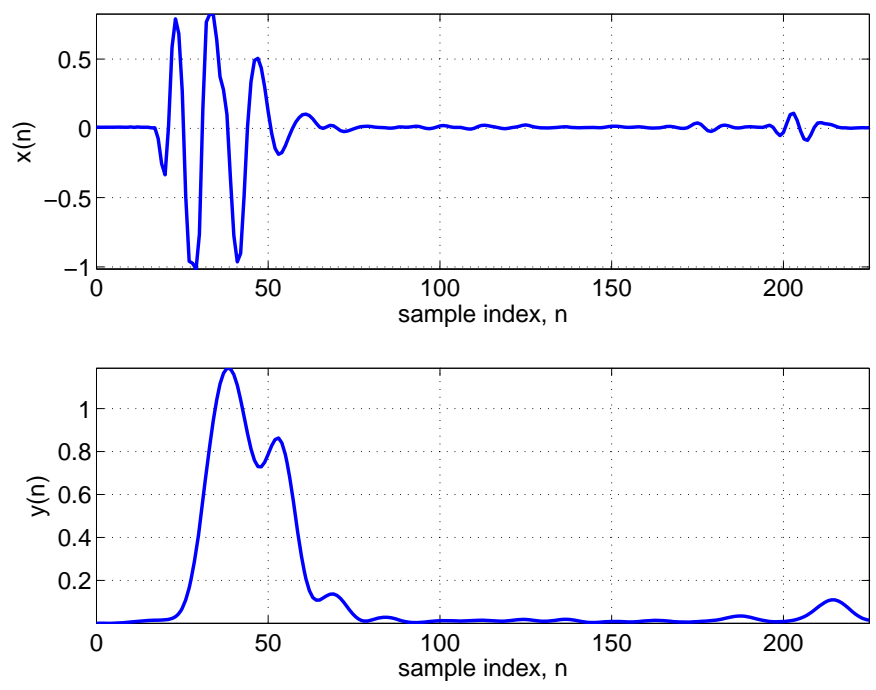

Fig. 1. Top: Sampled ultrasound pulse. Bottom: Result of applying the envelope detector in Eq. (3) and the low-pass filter to the pulse. The duration of the pulse corresponds to the thickness of the mill shell, which in this case was approximately $15 \mathrm{~mm}$.

\section{EXPERIMENTS}

Fig. 2 shows the experimental setup, with the water jet ultrasonic system mounted at the top left. A counter was mounted on the rotating shaft, giving 500 pulses per revolution, used to trigger the ultrasound measurement system. The drum is made of steel (grade 235JR) and has a diameter of $135 \mathrm{~mm}$, resulting in a circumferential scanning resolution of approximately $0.87 \mathrm{~mm}$. The circumferential velocity of the drum was approximately $1.75 \mathrm{~ms}^{-1}$. The thickness of the drum is $15 \mathrm{~mm}$.

Four artificial defects were introduced to the cylinder. One $25 \mathrm{~mm}$ deep hole with a diameter of $0.8 \mathrm{~mm}$ was drilled from one side of the cylinder. Three $0.4 \mathrm{~mm}$ wide cracks were 
then introduced, with a depth of $1 \mathrm{~mm}, 2 \mathrm{~mm}$, and $4 \mathrm{~mm}$, respectively. Fig. 3 shows a photograph of these defects.

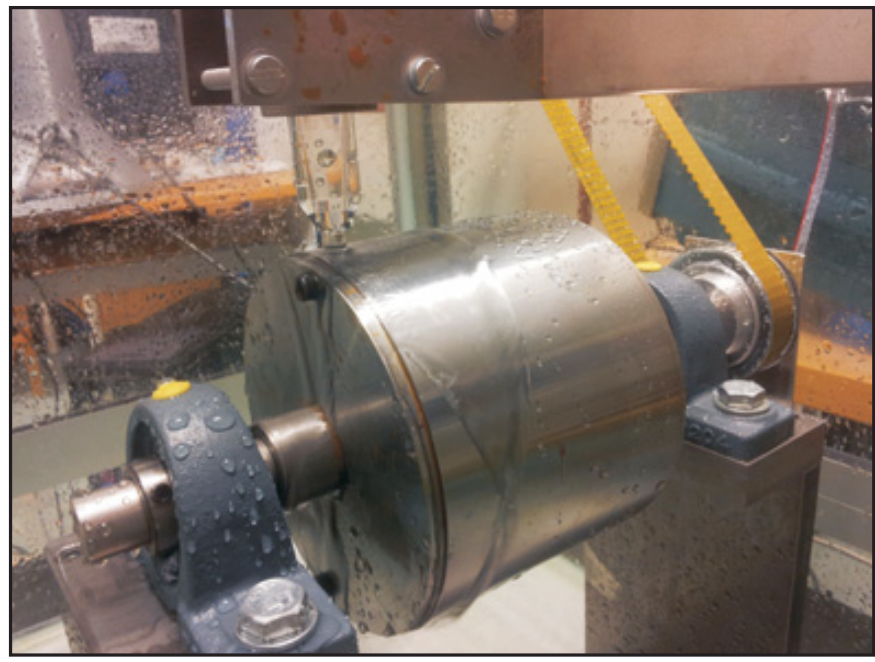

Fig. 2. Experimental setup with the rotating drum and the water nozzle mounted at the top left.

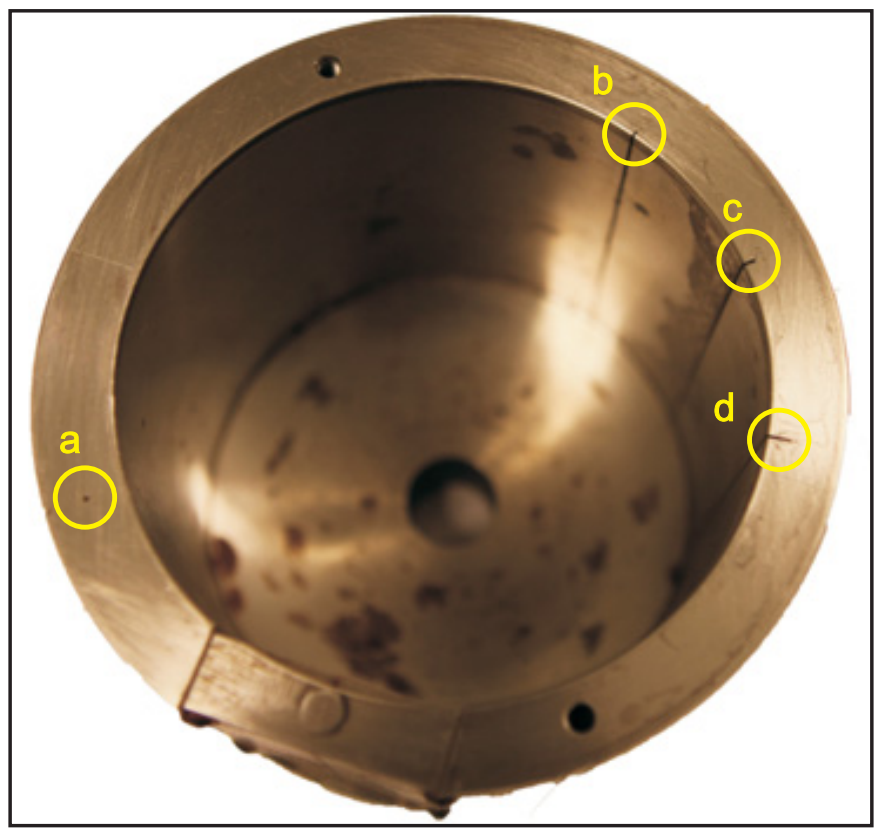

Fig. 3. Photograph of the artificial defects. Here (a) is a $25 \mathrm{~mm}$ deep hole with a diameter of $0.8 \mathrm{~mm}$, (b)-(d) are $0.4 \mathrm{~mm}$ wide cracks with depth $1 \mathrm{~mm}$, $2 \mathrm{~mm}$, and $4 \mathrm{~mm}$, respectively.

The ultrasound system was a H5KF Waterflow Probe from GE Inspection Technologies. The ultrasound transducer has a center frequency of $5 \mathrm{MHz}$ and a diameter of $10 \mathrm{~mm}$.

A step motor was then used to move the transducer for $60 \mathrm{~mm}$ along along the rotation axis, with a $1 \mathrm{~mm}$ step. As a result, $500 \times 60$ pulse-echoes (A-scans) were captured with an ADQ214 data acquisition card from SP Devices, Linköping, Sweden, working at a sampling rate of $200 \mathrm{MS} / \mathrm{s}$ and and a resolution of 14 bits. The measurements were then transfered to a computer for off-line processing using MATLAB.

Fig. 4 shows the schematics of the setup.

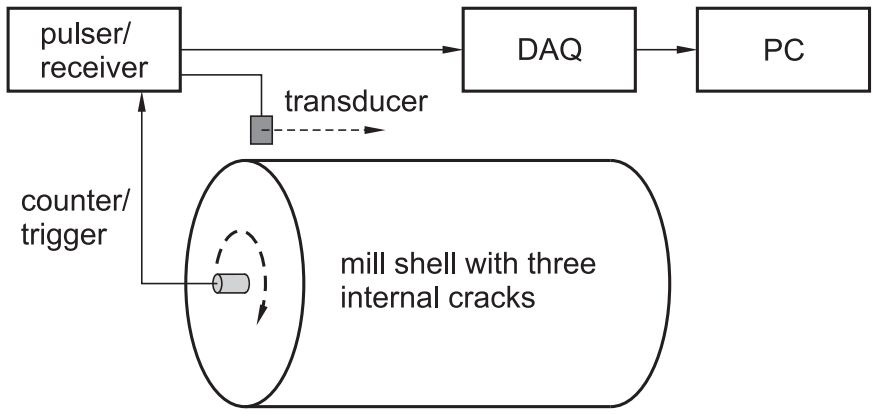

Fig. 4. Schematic picture of the entire setup.

\section{REsults}

After the scanning of the test sample was completed, the filtered envelope of each signal was computed as described in Section II-B. Using the amplitude of the pulse envelope as the color scale, an image can be formed showing the relative amplitudes of the reflected ultrasound pulse as a function of depth through the mill shell, for each point on the mill shell surface. Fig. 5 shows the result of this, for a section of the mill shell containing the defects. In the figure, bright colors correspond to stronger reflected ultrasound signals, while darker colors correspond to weak ultrasound signals.
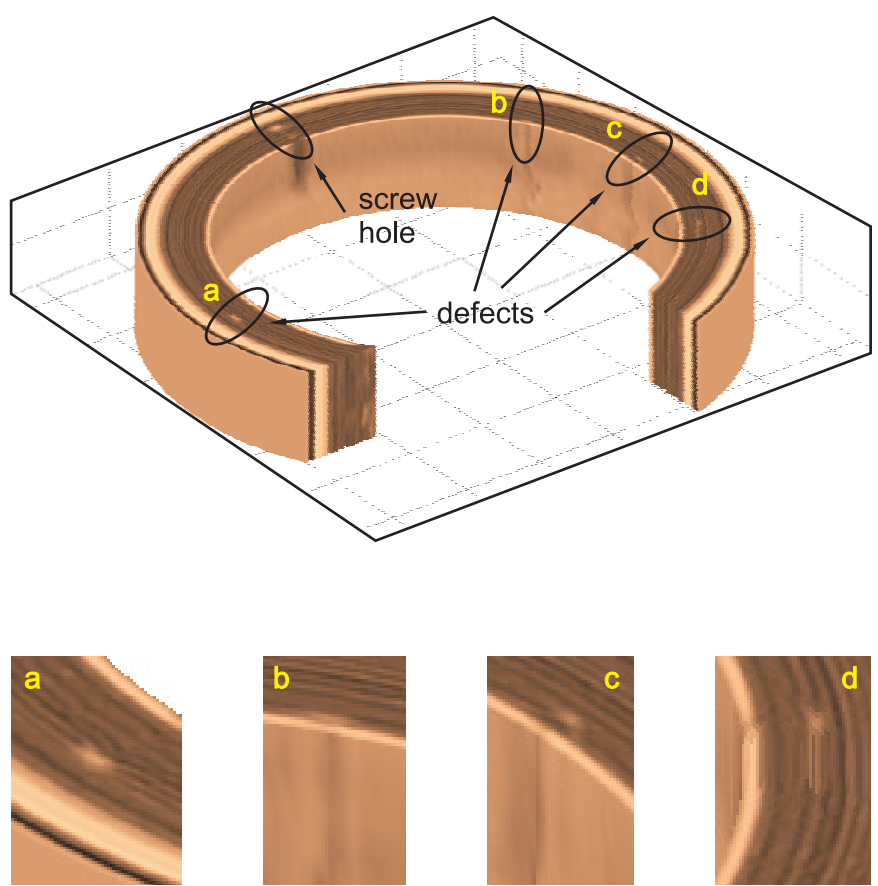

Fig. 5. Result of scanning the mill shell for internal defects. The defects are: (a) $25 \mathrm{~mm}$ deep hole with a diameter $0.8 \mathrm{~mm}$, (b) $1 \mathrm{~mm}$ deep crack, (c) $2 \mathrm{~mm}$ deep crack, (d) $4 \mathrm{~mm}$ deep crack. All cracks had a width of $0.4 \mathrm{~mm}$.

All four defects shown in Fig. 3 are also visible in Fig. 5. An artifact of only using the C-scans (no imaging algorithm 
applied to the data) is that the area beneath the defect will be shadowed, thus resulting in the illusion that there is also a defect on the inner surface of the mill shell. The top and bottom surface of the mill shell, and the top of the defects thus cause stronger backscattered echoes. Since no or little sound energy propagates through the actual defect, the area beneath it will be shadowed. This phenomenon could potentially be overcome to some extent by using a synthetic aperture focusing technique (SAFT) [12], but this is beyond the scope of the present study.

\section{Discussion}

In this paper only preliminary processing of ultrasound data has been performed, in the form of envelope detection and a simple low-pass filtering of the envelope. Furthermore, this pre-processing was not optimized in any sense in order to detect and classify flaws, but rather to facilitate visualization, as shown in Fig. 5.

The diameter of the water jet limits the aperture of the transducer, effectively to the diameter of the water jet. The aperture is, however, still significantly larger than the scanning step (the spatial scanning resolution). This means that some additional improvements of the imaging of defect could be made by applying, for example, a synthetic aperture focusing technique (SAFT) [12].

In the laboratory tests, the thickness of the mill shell was only $15 \mathrm{~mm}$, which is smaller than that of an actual mining mill $(20-30 \mathrm{~mm})$. For a thicker shell, the attenuation of a $5 \mathrm{MHz}$ ultrasound pulse will potentially be too large. If that is the case, changing to a transducer with lower center frequency will be appropriate, at the cost of a slightly lower imaging resolution.

The final quality of the system depends on several factors that should be investigated further in future work. For example, the distance from the sensor nozzle to the mill shell and the flow rate of the water jet strongly affect the results. In this study, these have been tuned experimentally, but the sensitivity of the systems to these settings have not been investigated.

\section{CONCLUSION}

In this study we have showed that the design of a water jet ultrasound scanning system for condition monitoring of mining mills is feasible, given the desired spatial resolution and the circumferential velocity of the mining mill.

We have also shown, in a laboratory-scale experiment, that small internal defects can be detected within a material with similar characteristics as the mining mill.

\section{ACKNOWLEDGMENT}

The authors would like to express their sincerest gratitude to Dr. Johan Borg, Luleå University of Technology, for his assistance and advice regarding the experimental setup, and to Dr. Matti Rantatalo for valuable discussions.

\section{REFERENCES}

[1] H. Kaczmarek, "Ultrasonic detection of damage in CFRPs," Journal of Composite Materials, vol. 29, no. 1, pp. 59-95, 1995.

[2] G. Marsh, "Finding flaws in composites," Reinforced Plastics, vol. 46, no. 12 , pp. 42-46, 2002.

[3] X. E. Gros, "Current and future trends in non-destructive testing of composite materials," Annales de Chimie Sciences de Materiaux, vol. 25, no. 7, pp. 539-544, 2001

[4] H. Berger, "Ultrasonic imaging systems for nondestructive testing," $J$. Acoust. Soc. Am., vol. 45, no. 4, pp. 859-867, 1969.

[5] R. J. Ball and D. P. Almond, "The detection and measurement of impact damage in thick carbon fibre reinforced laminates by transient thermography," NDT \& E International, vol. 31, no. 3, pp. 165-173, 1998.

[6] J. Lundberg, A. Bohlin, and M. Syk, "Blindfold tests on manganese crossing in railway application," Int. J. of Systems Assurance Engineering and Management, vol. 2, no. 2, pp. 169-182, 2011.

[7] — "Capacity test of ultrasonic equipment used for crack detection in railway application," Int. J. of Systems Assurance Engineering and Management, vol. 2, no. 2, pp. 183-192, 2011.

[8] V. Konshina and G. Dymkin, "Modern approaches to the certification of ultrasonic testing techniques," Russian Journal of Nondestructive Testing, vol. 44, no. 2, pp. 77-85, 2008.

[9] R. Dandotiya, J. Lundberg, and A. Wijaya, "Evaluation of abrasive wear measurement devices of mill liners," International Journal of COMADEM, vol. 14 , no. 2, pp. 3-17, 2011.

[10] NDT Resource Center, "UT material properties [homepage of NDT resource center]," July 282011.

[11] J. G. Proakis and M. Salehi, Communication Systems Engineering. New Jersey: Prentice Hall, 2012

[12] J. A. Seydel, Research Techniques for Nondestructive Testing. London: Academic Press, 1982, vol. 3, ch. Ultrasonic Synthetic-aperture Focusing Techniques in NDT, pp. 1-47. 